\title{
Grau de percepção e incômodo quanto à condição facial em indivíduos com paralisia facial periférica na fase de seqüelas
}

\author{
Degree of perception and discomfort regarding facial condition in subjects \\ with peripheral facial paralysis in sequelae stage
}

\author{
Kátia Cristina Silva de Freitas¹, Maria Valéria Goffi Gómez²
}

\begin{abstract}
RESUMO
Objetivo: Correlacionar a auto-avaliação da condição facial do paciente, o grau de incômodo quanto às seqüelas e de prejuízo em atitudes diárias com os dados encontrados na avaliação fonoaudiológica. Métodos: Participaram da pesquisa 29 indivíduos, de ambos os sexos, média de idade de 46 anos, com média de 5,9 sessões de fonoterapia. Realizou-se avaliação fonoaudiológica da simetria e da movimentação da face e verificou-se a presença de sincinesias e contraturas por meio de instrumento publicado e padronizado. Além disso, realizou-se uma entrevista por meio de perguntas fechadas que permitiram a graduação da opinião do paciente quanto à sua própria face e influência desse problema em suas atividades sociais e profissionais. Resultados: Encontrou-se concordância quanto à auto-avaliação do paciente e a avaliação fonoaudiológica $(\mathrm{p}=0,0029)$, porém essa correlação não esteve presente em pacientes com menos de três sessões de fonoterapia. Não houve correlação significante entre o grau de sincinesia/contratura e o grau de incômodo referido pelo paciente, assim como o prejuízo em atividades sociais e profissionais não foi associado ao grau de paralisia, sincinesia, ou contratura. No entanto, houve correlação fraca com tendência à significação (r=-0,3250/p=0,085) quando comparou-se a autoavaliação do paciente com o grau de prejuízo referido. Conclusões: A autopercepção da condição facial nem sempre é concordante entre o profissional e o paciente, sendo que essa concordância aumenta em pacientes com maior tempo de terapia. Por outro lado, o impacto da condição facial na vida do paciente parece não depender do grau das seqüelas.
\end{abstract}

Descritores: Paralisia facial/psicologia; Sincinesia; Contratura; Impacto psicossocial; Relações interpessoais; Qualidade de vida/ psicologia; Perfil de impacto da doença; Auto-avaliação/psicologia

\section{INTRODUÇÃO}

A Paralisia Facial Periférica consiste na paralisia da mímica facial uni ou bilateral, decorrente de uma lesão do sétimo nervo craniano. Pode surgir por origem idiopática, traumática, iatrogênica, infecciosa, tumoral, congênita, metabólica ou tóxica ${ }^{(1-2)}$. O acometimento do sétimo nervo causa hipotonia dos 23 músculos faciais inervados por ele, e toda a hemiface ipsilateral à lesão torna-se comprometida em maior ou menor grau de acometimento ${ }^{(1,3)}$.

Trabalho realizado na divisão Clínica Otorrinolaringológica do Hospital das Clínicas da Faculdade de Medicina da Universidade de São Paulo - USP São Paulo (SP), Brasil, financiado por bolsa de aprimoramento da Fundação do Desenvolvimento Administrativo - FUNDAP.

(1) Aprimoramento pelo programa de Fonoaudiologia Clínica do Hospital das Clínicas da Faculdade de Medicina da Universidade de São Paulo - USP - São Paulo (SP), Brasil; Fonoaudióloga da prefeitura de Mairinque (SP), Brasil.

(2) Doutora, Fonoaudióloga do Hospital das Clínicas da Faculdade de Medicina da Universidade de São Paulo - USP - São Paulo (SP), Brasil.

Endereço para correspondência: Kátia Cristina Silva de Freitas. R. Patápio Silva, 191/112, Pinheiros, São Paulo - SP, CEP 05436-010.

E-mail: ka_freitas@yahoo.com.br

Recebido em: 15/9/2007; Aceito em: 11/3/2008
As seqüelas são descritas na literatura como inerentes à regeneração neural porque a recuperação das fibras nervosas geralmente ocorre de forma supranumerária, desviada, com falhas na transmissão entre axônios adjacentes ou hiperexcitabilidade nuclear ${ }^{(4-5)}$.

$\mathrm{Na}$ fase de seqüelas, geralmente observa-se contratura de face com ausência de linhas de expressão na testa, olho mais estreito, elevação da comissura labial e asa nasal, além de rima nasolabial mais pronunciada no lado comprometido e filtro desviado para este lado. Também podem ser observados movimentos incompletos e sincinesias nos movimentos de testa, olhos, nariz e lábios, assim como lacrimejamento excessivo durante atividades como a mastigação ${ }^{(6)}$.

As sincinesias, descritas na literatura como as seqüelas mais freqüentes, são caracterizadas pelo movimento involuntário que aparece em associação a um movimento voluntário de grupos musculares independentes. Dentre os tipos de sincinesia pode-se citar o fechamento involuntário dos olhos com o sorriso e a sincinesia oral, que origina movimentação da região da boca acompanhada de movimento voluntário de testa ou olhos ${ }^{(4,7)}$.

Muitos pacientes apresentam dificuldade para perceber suas sincinesias ${ }^{(7)}$. Geralmente, percebem que há algo errado 
com seus movimentos faciais apenas quando observam uma reação desaprovadora do interlocutor ao realizarem movimentos anormais durante a expressão facial, e acabam involuntariamente criando providências para prevenir distorções, o que resulta na fixação da face em uma aparência congelada. Como resultado, piscar, comer, falar e sorrir pode resultar em um movimento de massa desconfortável que reduz drasticamente a qualidade de vida ${ }^{(8)}$. Desse modo, as sincinesias tornam-se incômodas, pois distorcem os movimentos faciais e expressões, e podem resultar em contraturas e comprometimento da simetria facial em repouso ${ }^{(9)}$.

A contratura é outra seqüela freqüente, que ocorre pela somatória de vários fatores durante o crescimento das fibras do nervo facial. Ela pode ser definida como a rigidez observada na hemiface comprometida, ocasionada pelo brotamento axonal que leva à inervação supranumerária e perda da relação da unidade motora, além de resultar no encurtamento das fibras musculares $^{(6)}$.

Os pacientes com sequiela de uma paralisia facial de longa duração geralmente referem problemas de comunicação. Esses pacientes mostram uma face ambígua ou com tendência a má interpretação do estado emocional. Sabe-se que a expressão facial é importante para a comunicação e o auto-reconhecimento, fundamental para o senso de identificação e habilidade de integração social, uma vez que a face é a primeira forma de reconhecimento do ser humano ${ }^{(10-12)}$.

Com isso, a deformidade facial e os eventos involuntários e indesejáveis, comuns na fase de seqüela, mais do que prejudicar a estética e função, podem interferir na comunicação interpessoal, o que limita a expressividade do indivíduo e acarreta uma variedade de problemas psicossociais, como a depressão, ansiedade, rejeição e paranóia ${ }^{(13)}$. Algumas pesquisas constataram sintomas depressivos em $65 \%$ dos pacientes, o que mostra maior tendência à depressão nesses indivíduos do na população normal ${ }^{(11)}$.

Muitos estudos avaliam o impacto que a nova condição facial tem na qualidade de vida desses indivíduos. Encontrouse em um deles, diminuição da função social, graduada pelo SF-36 Health Survey Questionnaire, e maior prejuízo nos movimentos associados à expressão facial, como presença de sincinesias e menor movimento voluntário, ao avaliarem pacientes com seqüelas de paralisia facial que se classificaram como não efetivos para expressar emoções ${ }^{(10)}$.

Outros estudos pesquisam a qualidade de vida de pacientes com paralisia facial após remoção do neurinoma do acústico ${ }^{(14)}$. Observou-se que os pacientes com baixa auto-estima, menos idade e do sexo feminino, tinham mais tendência a uma menor qualidade de vida. Segundo o autor, o grau de impacto na qualidade de vida não depende do grau da paralisia. Outra pesquisa ${ }^{(15)}$, por sua vez, encontrou qualidade de vida comprometida em 50\% dos pacientes que avaliou, mas não conseguiu encontrar relação entre baixa qualidade de vida e idade, sexo, fatores cirúrgicos ou grau de paralisia.

Recentemente, têm crescido a preocupação com a satisfação, o desempenho funcional e a qualidade de vida do paciente com paralisia facial. A extensão desses comprometimentos é reconhecida pela American Medical Association Guides to the Evaluation of Permanent Impairment ${ }^{(7,10)}$.
Alguns autores, a fim de medir o grau de satisfação do paciente com relação à condição facial, para melhor tratar esses pacientes, têm comparado a auto-avaliação do paciente com a avaliação clínica do profissional. Ressaltam que freqüentemente são encontradas discordâncias entre a percepção da condição facial do ponto de vista médico e do paciente, além de haver, por vezes, discrepâncias entre a autopercepção do paciente e seu quadro clínico, o que pode influenciar nas decisões médicas, como intervenções cirúrgicas, e dificultar o processo terapêutico. Esses autores sugerem que a autoavaliação do paciente deve ser levada em conta na hora de definir os pontos a serem trabalhados em terapia, assim como são importantes a orientação e autoconhecimento do paciente quanto à sua face ${ }^{(10,16)}$.

Estudos têm relatado os benefícios proporcionados pela terapia neuromuscular e observado que a reabilitação composta por exercícios específicos, modulação sensorial somada a monitoramento visual, e a conscientização, auxilia na melhora dos movimentos faciais e diminuição das sincinesias, mesmo nos casos de paralisia de longa data ${ }^{(7,9)}$. Ressalta-se a terapia com exercícios miofuncionais somados ao biofeedback oferecido pela eletromiografia $(E M G)^{(11)}$. Segundo alguns autores, essa técnica se faz importante porque os receptores sensoriais intrínsecos musculares são poucos ou ausentes na musculatura facial. Com isso, há pouca informação estática e dinâmica na musculatura facial, o que faz com que os indivíduos apresentem apenas uma vaga idéia da ação de seus músculos faciais ${ }^{(7)}$.

A terapia miofuncional também tem contribuído para melhora dos sintomas de contratura facial. Autores encontraram menor queixa de contratura em pacientes que estavam em processo de reabilitação quando comparados a pacientes sem intervenção terapêutica ${ }^{(17)}$.

Portanto, a terapia fonoaudiológica na fase de sequielas é uma importante ferramenta que visa à reabilitação da motricidade facial e a integração social do paciente por meio da reprogramação do controle voluntário da musculatura facial, o que habilita o indivíduo a manipular os eventos indesejáveis comuns nessa fase ${ }^{(6)}$.

No entanto, para que a reabilitação miofuncional seja bem sucedida é preciso que haja um envolvimento ativo do indivíduo, pelo fato de que para compensar a regeneração aberrante do nervo facial necessita-se que haja uma reorganização neural, a qual ocorre somente com auxílio do sistema nervoso central por meio da autopercepção e autocontrole. Portanto, os candidatos à terapia devem estar motivados e apresentar adequada cognição e atenção ${ }^{(8,13)}$.

Com isso, os objetivos da pesquisa foram:

1. Comparar a auto-avaliação da condição facial do paciente com a avaliação clínica fonoaudiológica.

2. Correlacionar o grau de incômodo quanto às seqüelas (sincinesia e contratura) referido pelo paciente com o grau de seqüelas encontrado na avaliação clínica.

3. Correlacionar o grau de prejuízo em atividades diárias, referido pelo paciente, com o grau de comprometimento facial encontrado na avaliação clínica fonaudiológica. Assim como correlacionar esse grau de prejuízo com o grau da auto-avaliação do paciente. 


\section{MÉTODOS}

Este trabalho foi submetido e aprovado pela comissão de Ética e Pesquisa da instituição de origem (protocolo $\mathrm{n}^{\circ}$. 909/06).

Os critérios para seleção da amostra foram: ausência de comprometimento cognitivo ou sensorial que dificultariam a compreensão da entrevista; ausência de comprometimentos faciais co-mórbidos à paralisia facial (cirurgia plástica, por exemplo); e ausência de paralisia facial bilateral.

Participaram deste estudo prospectivo, 29 indivíduos, com idade entre 11 e 79 anos (média $=46$ anos), 12 do sexo feminino e 17 do sexo masculino, diagnosticados com paralisia facial periférica em fase de seqüelas, segundo dados do prontuário fonoaudiológico.

Os indivíduos da amostra estavam em acompanhamento no ambulatório de Otorrinolaringologia, setor de Fonoaudiologia da instituição, com média de 5,9 sessões de terapia miofuncional individuais correspondentes à rotina do ambulatório, incluindo avaliação, orientação, e prescrição de exercícios e feedback por meio do uso do espelho durante a terapia e em casa.

Quanto à etiologia, 21 pacientes estavam diagnosticados com paralisia de Bell, dois com paralisia de origem iatrogênica, dois com etiologia de Ramsay Hunt, dois com etiologia traumática, um com Lyme e um com a Síndrome de MelkersonRosenthal.

A condição funcional da face foi avaliada pela pesquisadora segundo o Sistema de Graduação Facial ${ }^{(18)}$, por meio da observação da face em repouso (0-20) e na execução dos movimentos: elevação de testa, sorriso, bico, elevação do nariz e fechamento dos olhos. Quantificou-se o grau de movimentação (20-100) e das sincinesias (0-15). Obteve-se também uma nota de avaliação total, correspondente à nota de movimento com subtração da nota de repouso e de sincinesia.

A pesquisadora realizou, oralmente, uma entrevista com perguntas fechadas sobre a opinião do indivíduo com relação à sua face e o impacto causado nas atividades sociais e profissionais. As perguntas foram divididas em quatro itens:

1. Avaliação da face em repouso (graduada de 0 - péssima a 4 - ótima);

2. Avaliação da face ao movimentar a testa, olho, nariz e lábios (graduada de 0 - péssima a 4 - ótima);

Os itens 1 e 2 foram somados e originaram a nota total de auto - avaliação do paciente.

3. Presença de queixa e grau de incômodo por sincinesias e contraturas ( 0 - nenhum a 4 - muito)

4. Presença de prejuízo nas atividades sociais e profissionais e grau do mesmo (0 - nenhum a 4 - muito);

A avaliação da face e a entrevista foram realizadas no mesmo dia, no próprio ambulatório, após autorização fornecida pelo paciente e seus responsáveis legais no Termo de Consentimento Livre e Esclarecido. A duração média da avaliação e entrevista foi de 20 minutos e não apresentou risco ou incômodo ao sujeito.

Foi realizado um corte na amostra com a finalidade de se comparar o grau de percepção do paciente quanto à sua condição facial, conforme o número de sessões realizadas.
Formaram-se dois grupos: indivíduos com três sessões ou menos; e indivíduos com oito ou mais sessões de terapia.

Após a coleta do material, os dados foram organizados em planilhas e submetidos à análise estatística pertinente, a qual se utilizou da correlação de Spearman para que fosse possível a comparação dos dados obtidos, utilizando-se o nível de significância $\mathrm{p}<0.05$, sugerido para estudos biológicos.

\section{RESULTADOS}

Houve uma correlação estatisticamente significante entre a auto-avaliação total do paciente e a avaliação geral fonoaudiológica $(r=0,41, p=0,029)$, o que sugere uma concordância entre a visão do paciente e do profissional quanto ao grau de comprometimento da face (Tabela 1).

Tabela 1. Avaliação clínica comparada à auto-avaliação total do paciente

\begin{tabular}{lcc}
\hline Correlação de Spearman & $\begin{array}{c}\text { Coeficiente de } \\
\text { correlação }(r)\end{array}$ & Significância (p) \\
\hline Ross total X Auto-avaliação & 0,41 & $0,029^{*}$ \\
$\begin{array}{l}\text { Ross total (3 sessões) X Auto- } \\
\text { avaliação }\end{array}$ & 0,177 & 0,624 \\
$\begin{array}{l}\text { Ross total (8 sessões) X Auto- } \\
\text { avaliação }\end{array}$ & 0,761 & $0,017^{*}$ \\
\hline
\end{tabular}

Legenda: ${ }^{*} p<0,05$; Ross total $=$ Avaliação total fonoaudiológica de todos os indivíduos; Ross total (3 sessões) = Avaliação total fonoaudiológica de pacientes que realizaram menos de 3 sessões de fonoterapia; Ross total ( 8 sessões) = Avaliação total fonoaudiológica de pacientes que realizaram mais de 8 sessões de fonoterapia

Ao relacionar o grau de incômodo quanto à sincinesia com o grau da sincinesia na avaliação fonoaudiológica, observou-se correlação muito fraca sem significância estatística $(r=0,26$, $\mathrm{p}=0,182$ ).

Quando se comparou o incômodo causado pela contratura com o grau desta em repouso, medido na avaliação fonoaudiológica, não se observou correlação ( $r=-0,23, \mathrm{p}=0,234)$, como mostrado na Tabela 2.

Tabela 2. Avaliação clínica da sincinesia e da face em repouso comparada ao grau de incômodo relatado pelo paciente

\begin{tabular}{lcc}
\hline Correlação de Spearman & $\begin{array}{c}\text { Coeficiente de } \\
\text { correlação }(r)\end{array}$ & Significância $(p)$ \\
\hline $\begin{array}{l}\text { Ross Sinc X Incômodo referido } \\
\text { pela sincinesia }\end{array}$ & 0,26 & 0,182 \\
$\begin{array}{l}\text { Ross Rep X Incômodo referido } \\
\text { pela contratura }\end{array}$ & $-0,23$ & 0,234 \\
\hline
\end{tabular}

Legenda: Ross Sinc = Avaliação fonoaudiológica quanto à sincinesia; Ross $\operatorname{Rep}=$ Avaliação fonoaudiológica quanto à contratura

Ao relacionar o grau de prejuízo com a nota da movimentação da face encontrada na avaliação fonoaudiológica, não foi observada correlação significante $(\mathrm{r}=-0,16, \mathrm{p}=0,409)$. Assim como não houve correlação com o grau da sincinesia $(\mathrm{r}=0,173, \mathrm{p}=0,370)$ nem com o grau de contratura em repouso $(\mathrm{r}=0,036, \mathrm{p}=0,852)$. 
Da mesma forma, o prejuízo não foi relacionado à condição geral da face, graduada na avaliação fonoaudiológica $(\mathrm{r}=-0,123, \mathrm{p}=0,525)$. Mas houve uma correlação fraca, com tendência à significância estatística entre a auto-avaliação do paciente e o prejuízo referido $(\mathrm{r}=-0,325, \mathrm{p}=0,085)$, como observado na Tabela 3.

Tabela 3. Grau de prejuízo comparado à avaliação clínica e autoavaliação do paciente

\begin{tabular}{lcc}
\hline Correlação de Spearman & $\begin{array}{c}\text { Coeficiente de } \\
\text { correlação }(r)\end{array}$ & Significância $(p)$ \\
\hline Ross Mov X Prejuízo & $-0,160$ & 0,409 \\
Ross Sinc X Prejuízo & 0,173 & 0,370 \\
Ross Rep X Prejuízo & 0,036 & 0,852 \\
Ross Total X Prejuízo & $-0,123$ & 0,525 \\
Auto-avaliação X Prejuízo & $-0,325$ & 0,085 \\
\hline
\end{tabular}

Legenda: Ross Mov = Avaliação fonouadiológica quanto ao movimento facial; Ross Sinc = Avaliação fonoaudiológica quanto à sincinesia; Ross Rep = Avaliação fonoaudiológica quanto à contratura; Ross Total = Avaliação total fonoaudiológica

\section{DISCUSSÃO}

No presente estudo, a correlação estatisticamente significante entre a auto-avaliação total do paciente e a avaliação geral fonoaudiológica $(r=0,41, p=0,029)$ sugere uma concordância entre a visão do paciente e do profissional, quanto ao grau de comprometimento facial.

Outros estudos ${ }^{(10)}$, ao comparar os escores do Sydney and Sunnybrook Systems e House-Brackmann com a auto-avaliação do paciente, também encontraram concordância nas duas avaliações. Foi observado um maior escore total na avaliação facial dos pacientes que se autoclassificaram efetivos para comunicaram seus estados emocionais, embora esta correlação não tenha sido significante. No entanto, a falta de movimentação de testa, encontrada na avaliação fonoaudiológica, foi correlacionada significantemente à percepção do paciente quanto ao impacto gerado em sua expressão facial, principalmente de surpresa e tristeza. Também houve diferença significante quando comparado o grau de sincinesia encontrado na avaliação fonoaudiológica com a percepção do paciente como não efetivo para expressar emoções. Com isso, os indivíduos que se classificaram não efetivos mostraram maiores graus de sincinesia, além de um menor movimento voluntário no sorriso e na expressão de surpresa e tristeza, segundo o Sunnybrook Facial Grading System.

Outro estudo ${ }^{(16)}$, por sua vez, avaliou a concordância da avaliação clínica e auto-avaliação realizada pelo paciente após a cirurgia de remoção do neurinoma do acústico. Utilizaram o Yanagihara System e House-Brackmann Grading System. Os escores encontrados pelos clínicos, no geral, concordaram significantemente com a auto-avaliação dos pacientes. No entanto, observaram algumas discrepâncias como o fato de pacientes completamente paralisados segundo o Yanagihara system e House-Brackmann não apresentarem sintomas compatíveis com os escores obtidos na avaliação médica. Concluíram que estas discrepâncias devem ser consideradas durante o processo terapêutico, e também ressaltaram que estes dois sistemas de avaliação (Yanagihara System e House-Brackmann Grading System) são adequados não só para o clínico como para o paciente, como instrumentos de auto-avaliação.

$\mathrm{Na}$ análise dos indivíduos com menos de três sessões de fonoterapia não se observou a correlação entre a auto-avaliação realizada pelo paciente e a avaliação fonoaudiológica $(r=0,177$, $\mathrm{p}=0,624)$. No entanto, na análise dos pacientes com mais de oito sessões fonoterápicas, observou-se correlação forte e estatisticamente significante $(r=0,761, \mathrm{p}=0,017)$. Deste modo, deduz-se que os pacientes com menos sessões têm mais dificuldade para perceber suas alterações faciais, o que torna necessário a elaboração de um programa de autopercepção sobre o estado facial desses indivíduos durante o processo terapêutico.

Pesquisadores ${ }^{(7)}$ também referiram a dificuldade de autopercepção das alterações faciais nos pacientes com seqüelas. No entanto, observaram redução das sincinesias com a reabilitação neuromuscular, e concluíram que a terapia miofuncional que engloba a percepção dos movimentos faciais, com o uso do feedback oferecido pela eletromiografia (EMG) juntamente com exercícios específicos, proporcionam informações proprioceptivas e cinestésicas: visual, auditiva ou combinada, que facilitam a autopercepção da condição facial e conseqüentemente, favorecem a reabilitação.

$\mathrm{O}$ resultado do presente estudo sugere que, embora haja uma correlação direta entre o grau de sincinesia e incômodo quanto maior o grau de sincinesia, maior o grau de incômodo -, essa correlação nem sempre é verdadeira $(\mathrm{r}=0,26, \mathrm{p}=0,182)$. Em alguns casos, classificados como grau leve pela avaliação fonoaudiológica, foram referidos sintomas de muito incômodo pelo paciente, assim como graus severos foram percebidos com pouco incômodo pelo paciente. Com isso, além da questão orgânica, há outros fatores que levam a um maior ou menor incômodo, como fatores psicossociais.

Ao contrário do presente estudo, encontrou-se uma pesquisa $^{(10)}$ que mostrou correlação entre o grau de sincinesia e a autoclassificação do paciente como ineficiente para expressar emoções, embora não tenha avaliado o respectivo grau de incômodo quanto à sincinesia. Ao avaliarem as sincinesias na expressão de felicidade, surpresa e tristeza pelo teste Sunnybrook Facial Grading System, notaram concordância entre o baixo desempenho no teste e a autoclassificação do paciente como não efetiva para expressar emoções. As sincinesias foram apontadas como sendo o maior problema durante a felicidade, uma vez que os músculos zigomático e orbicular dos olhos, relacionados a essa expressão, também são os mais envolvidos durante a sincinesia.

A falta de correlação do incômodo causado pela contratura com o grau desta, em repouso ( $\mathrm{r}=-0,23, \mathrm{p}=0,234)$, medido na avaliação fonoaudiológica sugere que a sensação de incômodo referida pelo paciente não depende apenas do grau da contratura, mas também de fatores individuais.

Outros estudos também graduaram a sensação de contratura, embora não tenham comparado o grau de incômodo com o de contratura avaliado clinicamente. Em um exemplo desse tipo de estudo ${ }^{(17)}$, os pacientes foram solicitados a graduar o 
incômodo quanto à contratura de 1 (sem contratura) a 5 (muita contratura) antes e depois de 10 sessões de terapia miofuncional. Encontrou-se uma média de 3,68 (pré-teste) e 3,54 (pósteste) no grupo que não realizou terapia miofuncional e 3,72 (pré-teste) e 2,37 (pós-teste) no grupo que realizou sessões de terapia. Essas notas mostram um incômodo médio, que decai em pacientes que realizaram terapia miofuncional.

O prejuízo em atividades diárias não foi relacionado às notas da condição facial graduada na avaliação fonoaudiológica $(\mathrm{r}=-0,123, \mathrm{p}=0,525)$. Porém, essa relação foi inversa, o que significa que houve uma tendência de os pacientes com melhor condição facial apresentarem menor nota para o prejuízo. A correlação fraca, com tendência à significância estatística entre a auto-avaliação do paciente e o prejuízo referido $(r=-0,325$, $\mathrm{p}=0,085$ ), sugere que pacientes que autoclassificaram a condição facial com valores baixos, apresentam maior tendência a ter prejuízo em suas atividades sociais e profissionais.

Os resultados da presente pesquisa sugerem que não há um tipo específico de seqüela que causa um maior impacto na qualidade de vida, mas ressalta a existência de uma variação individual. Alguns estudos que incluíram questionários abertos ressaltaram a importância dos sintomas psicossociais, os quais afetam a qualidade de vida desses pacientes e manifestam prejuízos funcionais ${ }^{(10)}$.

Na pesquisa referida anteriormente ${ }^{(10)}$, o comprometimento dos movimentos associados à expressão facial de emoções (falta de movimento e sincinesia) foi associado a uma menor qualidade de vida. Em pacientes que se classificaram como não efetivos para expressar emoções foi encontrado um menor escore social do que físico segundo o SF-36 Health Survey Questionnaire, o contrário do encontrado em pacientes que se autoclassificaram efetivos. Esse dado está de acordo com os achados do presente estudo no que se refere ao maior impacto social em pacientes que se autoclassificam como ineficazes.

Outra pesquisa ${ }^{(14)}$ utilizou-se dos testes Derriford Appearance Scale, COPE Questionnaire, Personal Report Questionnaire e Facial Paralysis Questionnaire para medir o grau de impacto na qualidade de vida de pacientes após a cirurgia para remoção do neurinoma do acústico. Não houve correlação estatisticamente significante entre o nível de impacto na qualidade de vida e o grau da paralisia facial, nem houve associação desse nível ao tempo de recuperação pós-cirurgia, o que concorda com o presente estudo. Por outro lado, o autor encontrou outros fatores que parecem influenciar na baixa qualidade de vida. Observou que as mulheres apresentaram maior impacto na qualidade de vida $(\mathrm{p}=0,02)$, assim como encontrou uma relação negativa significante entre a idade e o nível de impacto $(\mathrm{r}=-0,28, \mathrm{p}=0,005)$. Altos níveis de impacto também foram associados à baixa auto-estima $(\mathrm{r}=-0,59, \mathrm{p}=0,01)$. Esse fator foi referido como o mais importante, seguido da idade e sexo. Com isso, concluiu que considerar a opinião do paciente antes e depois da cirurgia e criar medidas para melhorar a autoestima e diminuir o impacto na qualidade de vida é importante, especialmente no grupo de risco.

Alguns autores ${ }^{(15)}$ avaliaram o impacto na qualidade de vida de pacientes após a cirurgia para remoção do neurinoma do acústico, decorridos cinco anos. Avaliaram-nos pela Facial Clinimetric Evaluation ( $\mathrm{FaCE}$ ) e compararam esses dados com a idade, sexo, fatores cirúrgicos (tamanho do tumor e tempo após cirurgia) e graduação da condição facial segundo o House-Brackmann. Observaram que 50\% dos indivíduos tinham função facial comprometida, sendo a média de comprometimento social igual a 67,1. O grau de impacto social predito pela $\mathrm{FaCE}$ não foi relacionado de forma estatisticamente significante com as variáveis idade, sexo, fatores cirúrgicos e graduação no House-Brackmann.

Ressalta-se que a diversidade entre os resultados das pesquisas sobre o tema discutido pode dever-se às diferenças metodológicas e aos diversos instrumentos utilizados. Esses achados incentivam a realização de novos estudos que aprofundem a discussão sobre a autopercepção e o incômodo quanto à condição facial dos pacientes com seqüelas de paralisia facial.

\section{CONCLUSÃO}

1. A percepção da condição facial nem sempre é concordante entre o profissional e o paciente, sendo que esta concordância aumenta em pacientes com maior tempo de terapia. Por isso, faz-se importante incluir no processo terapêutico estratégias de autopercepção sobre a nova condição facial.

2. O impacto da condição facial na vida do paciente parece não depender do grau das seqüelas, ou seja, o grau de incômodo com relação à sincinesia e/ou contratura, parece relacionar-se mais a fatores individuais do que ao grau obtido na avaliação clínica.

3. Poucos pacientes referiram apresentar prejuízo em suas atividades diárias e esse prejuízo não se relacionou à nota recebida na avaliação clínica, nem a um tipo de seqüela especificamente. No entanto, houve maior tendência de os pacientes que apresentaram uma auto-avaliação rebaixada, apresentarem queixa de prejuízo nas atividades sociais e profissionais.

Com isso, é importante considerar a opinião do paciente e criar estratégias de auto-avaliação para que as expectativas do mesmo e do terapeuta se aproximem, a fim de que sejam traçadas condutas terapêuticas mais efetivas. 


\begin{abstract}
Purpose: To correlate the self-evaluation of patients with facial paralysis regarding their facial condition, the level of sequelae discomfort and the negative consequences in daily activities, with the results found in clinical assessment. Methods: Twenty-nine subjects of both genders, with average age of 46 years and an average of 5.9 therapy sessions participated in this study. A clinical evaluation of facial symmetry and movement was carried out, verifying the presence of synkinesis and contractures using a facial grading system proposed by a standardized and published instrument. Moreover, an interview with closed questions was carried out, in order to measure the patient's opinion regarding how his own face influences his professional and social activities. Results: There was an accordance regarding the patient's self evaluation and the clinical assessment $(\mathrm{p}=0,0029)$, but this correlation was not found in patients with less than three sessions of the speech therapy. No significant correlation was found between the degree of synkinesis/contracture and the level of discomfort reported by the patient. Furthermore, the negative consequences in professional and social activities were not associated to the degree of facial paralysis, synkinesis or contracture. However, there was a weak correlation tending to significance $(\mathrm{r}=-0,3250 / \mathrm{p}=0,085)$ when the self evaluation was compared to the reported negative consequences. Conclusions: The patient's self perception of the facial condition is not always in agreement with the professional's assessment, although this agreement increases in patients with longer intervention periods. On the other hand, the impact of the facial condition in the patient's life does not depend on the degree of sequelae.
\end{abstract}

Keywords: Facial paralysis/psychology; Synkinesis; Contracture; Psychosocial impact; Interpessoal relations; Quality of life/ psychology; Sickness impact profile; Self assessment (Psychology)

\title{
REFERÊNCIAS
}

1. Bento RF, Barbosa VC. Paralisia facial periférica. In: Lopes Filho O, Campos CAH. Tratado de otorrinolaringologia. São Paulo: Roca; 1994. p. 888-911.

2. Valença MM, Valença LPAA, Lima MCM. Paralisia facial periférica idiopática de Bell: a propósito de 180 pacientes. Arq Neuropsiquiatr. 2001;59(3B):733-9.

3. Caropreso VR. Paralisia facial bilateral - causas e tratamentos [monografia]. São Paulo: Centro de Especialização em Fonoaudiologia Clínica; 2000.

4. Moran CJ, Neely JG. Patterns of facial nerve synkinesis. Laryngoscope. 1996;106(12 Pt 1):1491-6.

5. Bajaj-Luthra A, VanSwearingen J, Thornton RH, Johnson PC. Quantitation of patterns of facial movement in patients with ocular to oral synkinesis. Plast Reconstr Surg. 1998;101(6):1473-80.

6. Gomez MVSG, Vasconcelos LGE, Bernardes DFF. Reabilitação miofuncional da paralisia facial periférica. In: Ferreira LP, Befi-Lopes DM, Limongi SCO. Tratado de fonoaudiologia. São Paulo: Roca; 2004.

7. Brach JS, VanSwearingen JM, Lenert J, Johnson PC. Facial neuromuscular retraining for oral synkinesis. Plast Reconstr Surg. 1997;99(7):1922-31; discussion 1932-3. Comment in: Plast Reconstr Surg. 1998;101(2):554-5. Plast Reconstr Surg. 2003;111(7):2370-5.

8. VanSwearingen JM, Brach JS. Changes in facial movement and synkinesis with facial neuromuscular reeducation. Plast Reconstr Surg. 2003;111(7):2370-5. Comment on: Plast Reconstr Surg. 1997;99(7):1922-31; discussion 1932-3.

9. Nakamura K, Toda N, Sakamaki K, Kashima K, Takeda N. Biofeedback rehabilitation for prevention of synkinesis after facial palsy. Otolaryngol Head Neck Surg. 2003;128(4):539-43.
10. Coulson SE, O'dwyer NJ, Adams RD, Croxson GR. Expression of emotion and quality of life after facial nerve paralysis. Otol Neurotol. 2004;25(6):1014-9.

11. Byrne PJ. Importance of facial expression in facial nerve rehabilitation. Curr Opin Otolaryngol Head Neck Surg. 2004;12(4):332-5.

12. Heymans PG, Beurskens CH. Emotional readability of facial displays presented by patients with sequelae of facial paralysis. Psychosocial aspects of facial dysfunction. Otol Neurotol. 2002;23(Supp 1): S107.

13. Diels HJ, Combs D. Neuromuscular retraining for facial paralysis. Otolaryngol Clin North Am. 1997;30(5):727-43. Review.

14. Cross T, Sheard C, Garrud P, Nikolopoulos TP, O'Donoghue GM. Impact of facial paralysis on patients with acoustic neuroma. Laryngoscope. 2000;110(9):1539-42. Comment in: Curr Surg. 2005;62(2):156-61; quiz 161.

15. Lee J, Fung K, Lownie SP, Parnes LS. Assessing impairment and disability of facial paralysis in patients with vestibular schwannoma. Arch Otolaryngol Head Neck Surg. 2007;133(1):56-60.

16. Ikeda M, Nakazato H, Hiroshige K, Abiko Y, Sugiura M. To what extent do evaluations of facial paralysis by physicians coincide with self-evaluations by patients: comparison of the Yanagihara method, the House-Brackmann method, and self-evaluation by patients. Otol Neurotol. 2003;24(2):334-8.

17. Beurskens $\mathrm{CH}$, Heymans PG. Positive effects of mime therapy on sequelae of facial paralysis: stiffness, lip mobility, and social and physical aspects of facial disability. Otol Neurotol. 2003;24(4):677-81.

18. Ross BG, Fradet G, Nedzelski JM. Development of a sensitive clinical facial grading system. Otolaryngol Head Neck Surg. 1996;114(3):380-6. 